\title{
DEBATES
}

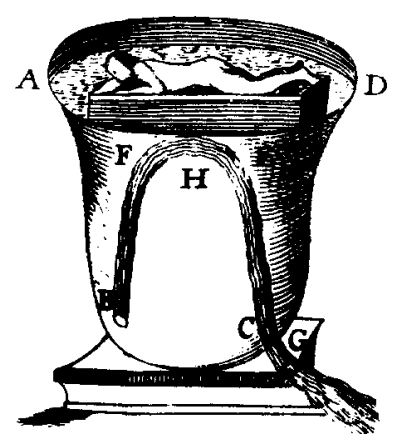

\section{ASALTO AL CASTILLO: ¿A QUÉ ESPERAMOS PARA ABORDAR \\ EN SERIO LA FORMACIÓN DIDÁCTICA DE LOS PROFESORES UNIVERSITARIOS DE CIENCIAS?}

CAMPANARIO, JUAN MIGUEL

Área de Didáctica de las Ciencias Experimentales. Departamento de Física. Universidad de Alcalá 28871 Alcalá de Henares. Madrid juan.campanario@uah.es

Resumen. Se plantea la necesidad de abordar la formación didáctica de los profesores universitarios de ciencias. Se discuten algunos enfoques inadecuados y algunas posibles alternativas que se estiman útiles.

Palabras clave. Formación de profesores, profesorado universitario, profesores de ciencias

Summary. The need for the pedagogical training of university professors of sciences is analysed. I discuss some inadequate approaches and some workable alternatives.

Keywords. Science university professors, university professors training. 
Las almenas de los torreones y los parapetos

demolieron y cuantos pilares allí los aqueos en el suelo estribaron cual firme sostén de sus torres. $Y$ tiraban de aquéllos queriendo arruinar la muralla. Mas los dánaos obstaculizaban su libre camino; reforzábanse los parapetos por medio de escudos $y$ desde ellos, al pie del bastión, a los otros herían.

Homero (La Ilíada, Canto XII)

\section{INTRODUCCIÓN}

El nuevo siglo trae nuevos retos para las universidades. Desde el ministerio correspondiente se plantea un debate sobre una nueva ley que regule el funcionamiento de los centros de enseñanza superior del futuro y uno de los objetivos básicos de dicha ley es mejorar la calidad de la docencia y estimular la competencia entre las universidades para conseguir los mejores alumnos. Ciertamente, la realidad social y económica de nuestro país ha cambiado radicalmente en los últimos años y va a seguir cambiando, con todo lo que ello implica para la enseñanza superior.

En lo que se refiere a la calidad de la función básica de la universidad, la docencia, existe un debate abierto sobre el tema. Por ejemplo, es frecuente encontrar en la prensa quejas que denuncian el uso abrumador que se hace en nuestras aulas de métodos docentes basados casi únicamente en la clase expositiva. En este contexto de cambio normativo, parece que éste es el momento políticamente adecuado para plantear nuevas exigencias al profesorado universitario.

En esta tesitura, el área de conocimientos de didáctica de las ciencias tendrá que acabar por hacer frente a un problema que, en muchos sentidos, también es una oportunidad: la impostergable necesidad de abordar una formación didáctica del profesor universitario de ciencias. Éste es un reto común también a otras didácticas específicas y un tema en el que la labor realizada ha sido escasa y adolece de falta de continuidad.

Cruz ha revisado la breve y tortuosa historia de la formación pedagógica del profesor universitario en España (Cruz, 2000). Según esta autora, las primeras propuestas para la formación pedagógica de los docentes universitarios se plantean a finales de los años ochenta, si bien pueden encontrarse algunos antecedentes previos que han tenido escasa incidencia. En los años noventa cuando, la evaluación del profesor universitario, primero a partir de las encuestas a los alumnos y, más adelante, en el contexto de la evaluación institucional de la calidad de la universidad, comienza a asumirse que «la formación pedagógica del profesor universitario, entre otras variables, es un medio adecuado para asegurar la calidad de la enseñanza y hacer posible su mejora si se estima necesaria» (Cruz, 2000, p. 22). Aunque desde entonces no son escasos los congresos y las jornadas celebradas sobre esta problema (por ejemplo, www.uva.es/aufop/ publica/revelfop/99-v2n1.htm), lo cierto es que este tema no suscita la atención que merece.

En nuestro caso, las implicaciones que tiene este problema se extienden al dominio de la enseñanza secundaria. En efecto, como señalan Cartaña y Comás, «la deficiente formación didáctica que tienen, en general, los profesores de ciencias de las facultades (y que, dicho sea de paso, no suelen reconocer) la han transmitido a sus alumnos (profesores de secundaria) y éstos, a su vez a sus alumnos» (Cartaña y Comás, 1994, p. 262).

En nuestro país, se constata un aparente abandono de este terreno por un área de conocimientos como la nuestra, más allá de algunos tímidos y loables intentos. Un indicio significativo ilustra lo asumida que tenemos esta pérdida sin lucha: un libro colectivo reciente, que pretende servir como elemento de referencia en el área de didáctica de las ciencias experimentales (Perales y Cañal de León, 2000), ni siquiera aborda el nivel universitario con una mínima profundidad en los capítulos destinados a plantear y analizar la formación del profesorado de ciencias. En 1993 Calatayud y Gil calificaban de «necesidad emergente» la preparación docente del profesorado de facultades de ciencias y, según parece, casi diez años después, todavía no hemos pasado al estadio de «necesidad abordada sistemáticamente».

El problema de la formación pedagógica de los profesores de ciencias en la universidad está, de antemano, mal condicionado. Es ésta la típica situación en la que existe una necesidad que no va acompañada del nivel de $d e$ manda que correspondería para su adecuada solución, por lo que cualquier intento de intervención debe pasar ineludiblemente por un esfuerzo previo de sensibilización de los posibles beneficiarios. Desgraciadamente, todo se complica por las actitudes negativas (a veces hasta la irracionalidad) que mantienen muchos posibles beneficiarios hacia nuestra área y nuestro trabajo de investigación (Campanario, en revisión).

Con este artículo pretendo dar un paso adelante en el planteamiento del problema de la formación didáctica del profesor universitario de ciencias, de manera que pase a constituir una prioridad en el área de conocimientos de didáctica de las ciencias. En este trabajo nos limitamos al análisis de la situación en España. En las secciones siguientes se analizan, en primer lugar, algunas de las características de la población a la que nos dirigimos. A continuación se enumeran algunos enfoques que, en principio, se estiman inadecuados para abordar el reto que ineludiblemente hemos de plantearnos. Se sigue con un análisis de los problemas previsibles y de las posibilidades de intervención, así como algunos logros que es razonable esperar conseguir en todo el proceso que se propone emprender. Por último, se sugieren algunas conclusiones y perspectivas. 


\section{SABEMOS POCO SOBRE QUIÉNES SON Y QUE PIENSAN LOS PROFESORES UNI- VERSITARIOS DE CIENCIAS}

El título de este apartado encierra toda una propuesta para una nueva línea de indagación. Cuando Porlán citaba las previsibles líneas de investigación futuras en didáctica de las ciencias, señalaba el desarrollo de una teoría del conocimiento profesional del profesor como una de ellas (Porlán, 1998). Sin embargo, a la hora de abordar la formación didáctica de los profesores universitarios de ciencias nos encontramos con un primer problema grave: en contraste con el número relativamente elevado de estudios centrados en los profesores de otros niveles, existe una escasez notable de investigaciones sobre esta población. Además, los pocos trabajos disponibles han abordado sólo algunas de las variables que inciden en el proceso de enseñanza-aprendizaje (Mellado, 1999, p. 234).

Dado que el conocimiento riguroso de los profesores universitarios, fruto de un proceso continuado de investigación, no existe, hemos de conformarnos con los escasos resultados publicados (Mellado, 1999), las impresiones personales o las conclusiones de los muy numerosos artículos periodísticos y de opinión que abordan directa o indirectamente el tema. Pasamos a describir algunas características generales.

En general, los profesores universitarios de ciencias no son demasiado entusiastas en lo que respecta a admitir la necesidad, por su parte, de una formación pedagógica. La percepción que se tiene de las áreas de conocimientos relacionadas con la didáctica no es muy positiva. Se tiende a considerar que las investigaciones sobre problemas de aprendizaje son más fáciles de realizar que las que se llevan a cabo en otros dominios y los resultados que se obtienen en ellas sólo son útiles cuando son directamente aplicables (Campanario, en revisión). Nuestra experiencia personal con la formación de profesores de secundaria es bien ilustrativa. En los cursos y asignaturas Técnicas de Evaluación Educativa o Comprensión y Elaboración de Textos que hemos tenido ocasión de impartir y que estaban orientados, en principio, tanto a licenciados de todas las áreas, con interés en dedicarse profesionalmente a la docencia, como a profesores de secundaria en formación (alumnos del CCP), hemos podido constatar cómo los procedentes de carreras científicas eran siempre los más reacios a seguir las recomendaciones didácticas. En particular, cuando se trataba de aplicar los conocimientos adquiridos sobre los procesos de comprensión de textos a la mejora de unidades didácticas, siempre hemos encontrado el mismo problema: los físicos y los químicos recién salidos de la universidad se centran, fundamentalmente, en los aspectos relacionados con la corrección científica y con la lógica de la disciplina (es decir, que no se cometan errores y que los contenidos se traten en el orden lógico, tradicional). $\mathrm{Mu}-$ chas veces resultaba vano todo el esfuerzo realizado para conseguir que los alumnos utilizasen los recursos disponibles con el fin de mejorar la comprensión de un libro de texto (por ejemplo, mediante títulos y subtítulos adecuados y otros recursos como marcadores explícitos).
Una de las concepciones docentes citadas por Calatayud y Gil en un estudio realizado con trenta y nueve profesores universitarios era la que concibe la enseñanza «como una actividad simple para la que bastan conocimientos científicos, sentido común, experiencia y algunos complementos sobre educación» (Calatayud y Gil, 1993, p. 36). Como acertadamente señala Beléndez, «el extenso uso que se hace de la lección unidireccional se debe a la rapidez y sencillez para la transmisión de conocimientos, pese a sus conocidos inconvenientes» (Beléndez, 1996, p. 192). Ciertamente, cuando se pregunta a los profesores universitarios, a éstos les resulta difícil encontrar otros ejemplos de profesiones que puedan aprenderse y desarrollarse con éxito tras un proceso de «formación» como el que se propugna para la propia, basado en la experiencia, el ensayo y el error, y la ausencia de capacitación reglada.

Debemos tener en cuenta, además, que muchos profesores universitarios de ciencias no distinguen ciertas diferencias entre nuestra área y otros dominios relacionados que a nosotros, expertos en el tema, nos parecen evidentes. A título de ejemplo, citaremos el hecho de que profesores de física que son perfectamente conscientes de las enormes diferencias que existen entre las distintas áreas de conocimiento en su disciplina todavía se sorprenden cuando confieso mi ignorancia en temas de investigación que parecen básicos en nuestra área (p.e., la resolución de problemas). Muchas veces parece que se tiene de nosotros una idea similar a la de un médico de familia, que, en principio, debe saber un poco de todo (y mucho de nada).

Mi experiencia profesional en el entorno de una facultad de ciencias me ha permitido detectar la ambivalencia que existe entre los profesores universitarios hacia la didáctica de las ciencias. Así, por ejemplo, no resulta raro que profesores que creen que pueden prescindir de cualquier formación didáctica y que consideran nuestro dominio como un terreno de investigación de segunda clase, se muestren cautos, e incluso reacios, a la hora de permitir que miremos sus exámenes, husmeemos en sus apuntes, fisguemos en sus clases teóricas o analicemos sus prácticas de laboratorio. Dado que estos profesores no tienen en gran estima nuestros conocimientos y capacidades, la prevención que sienten resulta sorprendente. Un compañero nos propuso, como ejemplo de algo similar, su actitud general hacia videntes y adivinos: «los considero unos charlatanes y no creo que puedan predecir mi futuro, pero no les dejo echarme las cartas del Tarot (por si acaso)».

Si extrapolamos los resultados de las investigaciones realizadas sobre los profesores de ciencias de enseñanza secundaria, hemos de situar, en el proceso de formación implícita al que han sido sometidos a lo largo de tantos años de haber sido alumnos, el origen de las ideas y las concepciones docentes de los profesores universitarios (Campanario, 1998a). Es ahí, sin duda, donde se han desarrollado sus concepciones acerca de la enseñanza y el aprendizaje de las ciencias. En ese contexto se originan también algunos mitos comunes en el imaginario de los profesores de ciencias que sirven como referentes y, 
en algunos casos, como modelos a seguir o a evitar. Así, por ejemplo, es omnipresente en casi todas las carreras la leyenda del profesor hueso con el que era dificilísimo aprobar (y, lógicamente, conseguirlo evidenciaba una capacidad intelectual fuera de toda duda), o aquella otra del profesor genio, muy bueno en su campo, pero que explicaba fatal (esto último a veces se considera un complemento indispensable del genio), así como otras historias y lugares comunes que ayudan a sus conocedores a sentirse miembros de una disciplina, un grupo, una escuela de pensamiento o un colegio invisible.

La conclusión inevitable de este apartado es, sin duda, que sabemos muy poco sobre los profesores universitarios de ciencias, que necesitamos investigar y aprender más sobre ellos, sus concepciones y hábitos docentes, sus actitudes y motivaciones y sus estrategias de enseñanza, no tanto para lamentar sus vicios o malos hábitos y llorar la falta de exigencia de una formación pedagógica específica como para averiguar el modo de acercarnos mejor a ellos.

\section{AL ABORDAR LA FORMACIÓN DIDÁCTICA DE LOS PROFESORES DE UNIVERSIDAD SE PUEDEN COMETER ERRORES}

En esta sección se analizan algunas posibles actuaciones que, a mi juicio, debemos evitar cuando intentemos abordar la formación didáctica de los profesores universitarios de ciencias. El lector interesado en otros problemas de tipo general puede consultar el excelente trabajo de Cruz (2000).

En los momentos actuales, un primer y evidente error sería hacer obligatoria (si ello fuese posible) cualquier tipo de formación didáctica. Además es dudoso que, en esta etapa de nuestra tarea, pudiésemos hacer efectiva dicha obligatoriedad; esta solución probablemente se traduciría en actitudes todavía más negativas hacia los posibles programas de formación. Sin embargo, hemos de convenir que, a medio y largo plazo, un objetivo estratégico básico debería consistir precisamente en conseguir que la formación pedagógica de los profesores universitarios de ciencias fuera un requisito necesario para acceder a la función docente, al igual que sucede en otros niveles. Cabe citar algunas razones que avalan dicha necesidad:

1) Los programas de formación voluntarios no se ajustan a ninguna regulación general que determine y organice los objetivos y contenidos. Es ésta una situación atípica similar a la de alguien que se siente enfermo y acude a una instancia que se autoproclama capacitada para curar y allí recibe una medicina que depende no tanto de su patología como del médico que lo atiende.

2) Como señala Cruz, en el contexto voluntario actual, «los profesores que asisten a las actividades de formación están altamente motivados por la calidad de su enseñanza» (Cruz, 2000, p. 25). Estos profesores están interesados por mejorar algo que en principio no hacen del todo mal. Esto significa que, hoy por hoy, estamos condenados a atender a los enfermos más leves.

3) Además, debemos evitar la tentación de caer en una formación didáctica limitada a cursos generales sobre educación, desconectados de los contenidos concretos y de la propia didáctica de las ciencias (Calatayud y Gil, 1993). La medicina debe ser específica y atacar las causas más que los síntomas. Una regulación eficaz puede conseguir esta especificidad.

Debemos evitar repetir errores y vicios propios de otras disciplinas, en particular, algunos hábitos importados de la física (terreno del que procede una buena parte de los investigadores en didáctica de las ciencias en nuestro país). Así, por ejemplo, es bien conocida la rivalidad que existe entre físicos teóricos y el resto del universo conocido (incluyendo otras especialidades de la física). Aun a riesgo de generalizar injustamente, se puede contrastar que muchos físicos teóricos se consideran una especie de casta aparte, lo cual se manifiesta incluso en su negativa a vestir la clásica bata de laboratorio. Algunos incluso se sienten orgullosos de la inutilidad (entendida como dificultad para la aplicación inmediata) de sus trabajos de investigación. También en nuestra área puede darse el caso de repetir este vicio y caer en la tentación de valorar más unas líneas de investigación supuestamente complicadas sobre otras más útiles de cara a la docencia directa. Un planteamiento así no hará sino confirmar, desde el punto de vista externo, la completa inutilidad y palabrería que suele achacarse a los que trabajamos para entender los procesos de aprendizaje.

También sería un error plantear nuestros esfuerzos de formación simplemente como un parche que pueda ayudar a mantener la situación actual o a resolver algún problema puntual del profesorado universitario sin cambiar realmente las formas de pensar y actuar. Un ejemplo importado de la enseñanza secundaria nos ayudará a apreciar mejor las consecuencias de un error así. Es bien sabido que la resolución de problemas es una tarea en la que se registra un grado elevado de fracaso en los alumnos. Ante esta solución caben muchas respuestas, una de ellas consiste en enseñar a los alumnos estrategias y técnicas para resolver problemas tradicionales y otra, más radical, consiste en cambiar de raíz la tarea que origina las dificultades y sustituir los problemas tradicionales por problemas abiertos y problemas como pequeñas investigaciones (Gil, Carrascosa, Furió y Martínez-Torregrosa, 1991; Gil, Martínez-Torregrosa y Senent, 1988). Igualmente, si la didáctica de las ciencias experimentales se dedica únicamente a taponar los (enormes) boquetes que se aprecian a simple vista, nuestro futuro será incierto una vez que el tapón haya sido puesto en su sitio y las cosas sigan como están. Así, por ejemplo, son discutibles líneas de actuación que consistan únicamente en implementar cursos sobre diseño de transparencias (sin más cambios), elaboración y defensa del proyecto docente (de cara a las típicas oposiciones universitarias) o sobre cómo hablar en público. Cada vez que admitimos que con un curso sobre uno de estos temas estamos abordando la capacitación 
didáctica de los profesores, desperdiciamos una oportunidad de oro, bendecimos una deficiente formación pedagógica y transmitimos ideas inadecuadas sobre nuestro trabajo y sobre el área de conocimientos de didáctica de las ciencias experimentales.

Por otra parte, debemos evitar enfrascarnos en temas puntuales, pero con una repercusión escasa en la docencia diaria. Sirva como ejemplo, la formulación de objetivos didácticos. En nuestra facultad, un grupo de alumnos realizó con nosotros un trabajo de análisis de los programas docentes de las distintas asignaturas de los estudios de licenciado en química y licenciado en medio ambiente. El estudio reveló que los programas estaban, en general, centrados en los contenidos y que, con frecuencia, faltaban los objetivos. Cuando éstos se incluían, solían estar mal formulados, muchos se confundían con actividades o aparecían enfocados al profesor (cosa lógica, si tenemos en cuenta que el profesor es el centro de la clase expositiva). Incluso en los proyectos docentes que se presentan en las oposiciones a las plazas de profesorado universitario, suelen aparecer objetivos mal formulados, sin que se distinga en muchos casos entre objetivos generales y específicos y, por supuesto, siendo desconocido el uso de taxonomías.

Formular objetivos siguiendo las reglas del método es una tarea compleja que, todo hay que decirlo, influye poco en la clase diaria del profesor. ¿Merece la pena empeñarnos en corregir este aspecto cuando existen otras necesidades más evidentes? Quizás debamos centrar nuestra atención en conseguir que el profesor sepa que existen otros objetivos diferentes a los puramente cognitivos y que estos objetivos son difícilmente alcanzables mediante la clase expositiva. No olvidemos, por otra parte, que en muchas ocasiones la relación lógica entre objetivos y contenidos se invierte y se preparan unos objetivos de acuerdo con unos contenidos previamente impuestos o seleccionados.

Es evidente también que no debemos plantear la necesidad de la formación pedagógica del profesor como algo que va a beneficiar únicamente a los alumnos. Algunos profesores universitarios de ciencias sospechan que en el área de didáctica de las ciencias experimentales tenemos vocación de defensores y valedores del alumno únicamente (Campanario, en revisión). No olvidemos que, en la situación actual de las universidades, uno de los temores que acechan al profesor es perder su autoridad tradicional y acabar trabajando en una jungla similar a aquélla en que se han convertido muchos centros de secundaria. En este contexto, es posible que cualquier cambio que se perciba como un beneficio real para los alumnos se asocie a una amenaza clara para el estatus del profesor.

Un último error que conviene evitar es el de ofrecer soluciones-milagro. La experiencia en didáctica de las ciencias demuestra que en nuestra área existen modas que se suceden con una cierta rapidez. Así, por ejemplo, tras el dominio abrumador de los puntos de vista conductistas, surgió el aprendizaje por descubrimiento para ser sustituido por nuevos enfoques que tienen en cuenta las ideas previas de los alumnos (Campanario y Moya, 1999). El área de didáctica de las ciencias es un terreno en el que existen muchas propuestas de investigación y actuación que provocan adhesiones masivas $y$, a veces, desencantos masivos. No podemos generar la impresión de que tenemos la solución a todos los problemas de enseñanza. Más bien, debemos insistir en la necesaria toma de contacto con dichos problemas como paso previo a la búsqueda de soluciones.

\section{LA FORMACIÓN DIDÁCTICA DE LOS PROFESORES UNIVERSITARIOS PLANTEA NUMEROSOS PROBLEMAS AL ÁREA DE DIDACTICA DE LAS CIENCIAS}

Si tenemos en cuenta que nos enfrentamos a un reto formidable, para el que existen escasos antecedentes, hemos de concluir que, tal vez, la relación de problemas que debemos plantear y resolver es abrumadora. En este apartado analizamos algunos de los que nos parecen más relevantes.

En primer lugar, como acertadamente señala Escudero, «con lo que nos encontramos al hablar de la formación permanente del profesorado universitario no es precisamente con un terreno deshabitado y deficiente que es preciso habitar y redimir mediante perspectivas, concepciones, procesos y prácticas centradas en la docencia y su mejora. Ya existen, más bien, otras perspectivas, normas y patrones, prácticas y relaciones, así como estructuras bien asentadas de regulación y recompensas, que habitan desde hace tiempo y de forma consistente dicho territorio» (Escudero, 1999, p. 136). Al igual que nuestros alumnos de ciencias llegan a nuestras aulas universitarias equipados con un arsenal de ideas previas o preconcepciones acerca de los contenidos científicos (amén de pautas de razonamiento y concepciones epistemológicas inadecuadas), hemos de admitir que los profesores universitarios han desarrollado, primero durante su época de estudiantes y luego durante su tarea docente diaria frente a la pizarra, ideas y concepciones sobre cómo es y cómo debe ser la docencia universitaria.

En segundo lugar, aunque cada vez es mayor tanto el interés por la formación didáctica del profesor universitario como la asistencia voluntaria a los programas de formación que se desarrollan en distintas universidades (Cruz, 2000), debemos enfrentarnos con la ineludible realidad de que nuestros servicios, en general, no son apreciados ni requeridos con mucho entusiasmo. Vamos a encontrar un problema actitudinal serio. Las razones son diversas y, aunque sería posible enumerar bastantes, no hay que olvidar que siempre es difícil admitir que uno no hace su trabajo tan bien como cree y tiene probablemente una parte de la «culpa» en las dificultades de aprendizaje de sus alumnos. También los médicos del pasado se resistían a aceptar que la falta de medidas de asepsia fuera la causa de la muerte de sus pacientes cuando ellos pasaban de las autopsias o de una intervención quirúrgica a atender otras intervenciones sin siquiera lavarse las manos. 
Estrechamente ligado con el problema anterior, otro obstáculo conceptual que va a exigir cierta dedicación quizás sea la intuición tan extendida de que los únicos problemas de enseñanza de las ciencias achacables al profesor universitario tienen que ver con la falta de conocimientos de la asignatura propia. Ésta es casi la única responsabilidad que muchos profesores de universidad están dispuestos a admitir (y no en ellos, naturalmente, sino en otros). Así, según este punto de vista, los problemas de enseñanza de la física o la química se solucionan... enseñando bien la física o química (bien significa enseñando la física o la química correcta, lo que equivaldría a enseñarla correctamente).

Otro problema estratégico consiste en determinar a qué población nos vamos a dirigir preferentemente. Aunque, en principio, pudiera pensarse que es mejor tratar de abordar únicamente la formación de los profesores universitarios noveles (ayudantes) y dar por perdidos a los profesores con más experiencia, esta decisión sería discutible por varias razones:

1)Los profesores noveles suelen estar muy ocupados con los estudios de doctorado, la elaboración de la tesis doctoral o el «engorde» de su currículo de cara a su futura estabilización laboral en la universidad.

2)Los profesores noveles suelen tener unas responsabilidades docentes limitadas muchas veces al desarrollo de clases de problemas y laboratorios. Un programa de formación didáctica no hará sino proporcionarles respuestas a preguntas que todavía no tienen ni sienten.

3)En línea con lo anterior, se necesita una cierta experiencia para poder detectar problemas de aprendizaje y enseñanza de las ciencias (p.e., persistencia de las preconcepciones o ideas previas de los alumnos).

4) Como consecuencia de su escasa experiencia docente, los profesores noveles suelen estar más ocupados por su propia actuación que por el aprendizaje de los estudiantes (Mellado, 1999, p. 237).

5)Por último, debemos evitar el peligro de que los profesores noveles acudan a nosotros atraídos fundamentalmente por el inevitable certificado que seguramente obtengan en nuestros cursos (el ejemplo de los cursos del CAP es suficientemente ilustrativo).

No hay que descartar, por tanto, dirigirnos también a profesores con más años de ejercicio siempre que estén dispuestos a asumir que todavía pueden influir en el aprendizaje de sus alumnos.

Sería un error pensar que las necesidades de formación de nuestros profesores universitarios de ciencias se pueden solventar con cursos en los que nos limitemos, fundamentalmente, a contar lo que hayamos descubierto como investigadores. Basta mirar revistas como Enseñanza de las Ciencias para comprobar que los temas de investigación en didáctica de las ciencias están alcanzando un nivel de especialización y detalle tal que, muchas veces, tienen una aplicación remota o escasa en una realidad tan necesitada de tratamientos globales como es la docencia en la universidad. Así, algo que decimos y proclamamos continuamente (la necesidad de aplicar o buscar aplicación de la investigación en didáctica de las ciencias a la realidad docente) puede que en el contexto en que nos movemos sea más un problema que una oportunidad.

Una dificultad adicional que debemos afrontar es la necesaria adaptación que hemos de hacer los expertos en didáctica de las ciencias a las concepciones epistemológicas de nuestros futuros beneficiarios para evitar cometer errores elementales. Una anécdota personal puede resultar ilustrativa. En nuestro departamento, el área de didáctica de las ciencias experimentales desarrolla, entre otras, una asignatura optativa denominada «Aprendizaje de las Ciencias Experimentales», orientada a los alumnos de química que tienen interés en dedicarse a la docencia en el futuro. Durante los primeros años de impartición de esta asignatura pretendíamos presentar muchas de las conclusiones sobre el aprendizaje basadas en la evidencia experimental que las respaldaba. Así, empleábamos una parte importante del tiempo analizando con los alumnos los experimentos que habían servido para obtener determinadas conclusiones y resultados. Entre otros aspectos analizábamos el diseño, las posibles limitaciones, la interpretación siempre complicada de los resultados, etc. No nos dábamos cuenta de que nuestros alumnos estaban acostumbrados a recibir en clase simplemente las conclusiones, las leyes y los principios de su disciplina, y no tanto la evidencia que las respalda. Durante nuestras explicaciones, los alumnos tomaban muy pocos apuntes, porque para ellos eran simples ejemplos las tareas que para nosotros eran fundamentales (el análisis crítico de los experimentos). Los estudiantes interpretaban estos experimentos-ejemplos como una ayuda para entender mejor unas cuantas conclusiones. Para ellos, la justificación de estas conclusiones era un problema inexistente, dado que, al igual que sucede en otras asignaturas de la carrera, ellos las suponían correctas y contrastadas. Como cualquier especialista en didáctica de las ciencias sabe, se requiere cierto tiempo para empezar a plantearse la necesidad de estas cuestiones. Igualmente, es posible que los profesores universitarios no estén tan interesados en conocer la fundamentación o el origen de las recomendaciones didácticas como a nosotros, especialistas, nos pudiera parecer en principio.

Debemos esperar muchas objeciones por parte de los profesores a las propuestas docentes que hagamos. Por tanto, otro problema que debemos abordar es la necesidad de ponernos en el lugar de los profesores en otros aspectos y tratar de entender sus argumentos (aunque no los compartamos). Ciertamente, hay argumentos que resultan difíciles de rebatir y contrarrestar. Además, estas objeciones se basan en actitudes negativas implícitas o explícitas. La realidad diaria en el contexto de una facultad de ciencias en la que muchos profesores consideran nuestra área como una extravagancia más de la universidad me ha permitido descubrir que los docentes universitarios son realmente hábiles encontrando $\mathrm{p}$ roblemas, inconsistencias, puntos débiles y posibles efectos secun- 
darios no deseados en casi cualquier idea o método docente alternativo que se somete a su consideración. En particular, será preciso combatir de manera creíble la tantas veces repetida afirmación de que en los niveles de enseñanza universitaria el alumno debería haber alcanzado ya un cierto nivel de madurez y, por tanto, no se necesita una formación didáctica del profesor. Además, se nos argumenta que los alumnos son pasivos y en este contexto la mayor parte de la responsabilidad recae en el profesor y consiste precisamente en explicar bien, y explicar bien quiere decir explicar según la lógica de la disciplina que, con el devenir de los siglos, ya está bastante bien estructurada. Según este punto de vista, el profesor, evidentemente, sólo necesita conocer bien su asignatura y el alumno sólo debe, lógicamente, captar la estructura de esa asignatura que le transmite el profesor. ¿Dónde hay aquí un hueco para la didáctica? Otra excusa contra nuestros propósitos es ésta: en el pasado siempre ha habido físicos y químicos sin que existiese formación didáctica de los profesores y sin que se sintiese la necesidad de la misma. Que estos y otros razonamientos sean inadecuados no nos exime de tenerlos en cuenta. Además, la refutación simple no siempre basta para eliminar estas objeciones.

\section{LA FORMACIÓN PEDAGÓGICA DE LOS PROFESORES UNIVERSITARIOS OFRECE NUEVAS POSIBILIDADES AL ÁREA DE DIDÁCTICA DE LAS CIENCIAS}

Un problema aparente puede, en realidad, ser una oportunidad, según se considere y según tengamos un margen mayor o menor de actuación. En este apartado analizamos algunas de estas posibilidades ligadas a la tarea que proponemos.

Un paso que no deberíamos obviar es, evidentemente, mirar a nuestro alrededor para identificar proyectos de formación didáctica de los profesores universitarios de ciencias con el fin de conocer aciertos y desaciertos. Así evitamos, sin duda, el peligro de repetir errores o de redescubrir la rueda. En nuestro propio país ya existen algunos programas de formación general de profesores universitarios que pueden servir como punto de partida (Cruz y Grad, 1993; Blázquez, 1999; Almajano y Valero-García, 2000). En otros países, por ejemplo en Latinoamérica, también se han puesto en marcha algunos planes de capacitación del profesorado universitario que han tenido un cierto éxito (www.uis.edu.co/programas/posgrados/cedeuis/index.html). En Colombia, incluso existe una revista llamada Docencia Universitaria con un marcado carácter multidisciplinar y con interesantes artículos de crítica junto con sugerencias concretas de actuación. Creemos que este tipo de intentos merece una atención detallada, habida cuenta de las dificultades propias del contexto en el que se llevan a cabo.

Cruz ha articulado una serie de propuestas generales con modelos (Cruz, 1993) y justificaciones razonadas y sensatas que deberíamos tener en cuenta a la hora de planificar cualquier proyecto de formación de profesores universitarios (Cruz, 2000). Además, dado que la formación didáctica del profesor universitario no es un problema exclusivo de nuestra área, podemos buscar aliados naturales en otras materias afines, como son la de didáctica de las matemáticas y la de didáctica de las ciencias sociales.

Quizás el reto más difícil, pero también el que ofrece más oportunidades, consista en determinar el conjunto mínimo de conocimientos y destrezas básicos que se estiman imprescindibles para desarrollar una docencia universitaria de calidad de acuerdo con nuestros conocimientos actuales en didáctica de las ciencias. Este conjunto mínimo formaría el núcleo inicial realmente imprescindible en cualquier proyecto de formación didáctica y, a partir de ahí, podrían derivarse módulos específicos sobre otros aspectos necesarios, pero menos urgentes. Independientemente de cual sea finalmente el conjunto mínimo de conocimientos y destrezas que se estiman necesarios como base didáctica del profesor universitario de ciencias, habría que dedicar un cierto tiempo a eliminar (o al menos cuestionar) algunos mitos comunes muy extendidos entre el profesorado universitario (p.e., la concepción de la mente del alumno como un recipiente pasivo vacío que hay que llenar, la supuesta eficacia más allá de toda duda de los trabajos prácticos, la idea de que las capacidades de los alumnos se distribuyen siguiendo una curva normal, etc. (Campanario, en revisión)). Como ejemplo de esta tarea desmitificadora, cabe citar un artículo reciente publicado en una revista ampliamente leída por los profesores universitarios de física que cuestionaba algunas otras evidencias de sentido común (Gil y Vilches, 1999).

Las preocupaciones anteriores no están de más: para aliviar la monotonía de las clases expositivas, los profesores universitarios pueden tratar de recurrir a otros enfoques tales como el uso masivo de los laboratorios de ciencias, la aplicación de ordenadores y otras tecnologías a la enseñanza o el recurso al aprendizaje por descubrimiento. Ni que decir tiene que estas falsas soluciones, utilizadas sin más y como única alternativa intuitiva a lo existente, pueden fracasar: Prácticas de laboratorio tradicionales, cerradas, con escasa participación intelectual del alumno, ordenadores utilizados sin una teoría acerca de la enseñanza y el aprendizaje y enfoques, como el aprendizaje por descubrimiento, ampliamente criticados desde diversos puntos de vista, no pueden sino empeorar la situación. Así pues, nuestra tarea se complica: tenemos que hacer ver al enfermo que estos remedios son peores que la enfermedad...

El asalto a esa fortaleza aparentemente inexpugnable que es la universidad puede proporcionar nuevos horizontes a la investigación en didáctica de las ciencias. Pensemos que, a diferencia de lo que sucede con los profesores de enseñanza secundaria, podemos dirigirnos a toda una población de científicos en ejercicio, que se dedican en cuerpo y alma a tareas de investigación en las más diversas áreas. Son enormes las posibilidades de realizar estudios sobre el profesorado universitario de ciencias, más allá de lo que se refiere a sus concepciones 
sobre la enseñanza y el aprendizaje y a la influencia que su contacto diario con la ciencia y la construcción del conocimiento científico ejerce en sus concepciones epistemológicas y en sus formas de razonar y entender, por ejemplo, las cuestiones de filosofía de la ciencia que, como es sabido, constituyen uno de los fundamentos de nuestra área de conocimientos (Gil, 1993; Mellado y Carracedo, 1993). Así, por ejemplo, hemos detectado la presencia de concepciones falsacionistas en los profesores universitarios de física, que, evidentemente, no se corresponden con la realidad de su trabajo diario, más orientado a confirmar teorías y modelos que a falsarlos. Podemos estudiar la evolución de sus puntos de vista tras el paso por nuestros programas de formación.

Tal vez una de las líneas de investigación prioritarias debería ser, precisamente, el estudio de las ideas y las actitudes de los profesores universitarios que sí sienten la necesidad y sí creen en la conveniencia de una formación pedagógica. El objetivo sería averiguar sus ideas y motivaciones para tratar de abrir una brecha en los muros de la fortaleza universitaria (estudiar al sano para curar al enfermo). Otro posible objeto de investigación podría ser nuestra propia área de conocimientos: todos somos físicos, químicos, biólogos y geólogos reconvertidos a didactas, ¿qué nos ha llevado a este cambio?, ¿cómo nos hemos interesado por este terreno de investigación y actuación?, ¿qué podemos aprender de nosotros mismos que nos resulte de utilidad para abordar la formación de los profesores universitarios de ciencias reacios a nuestra ayuda?

Además de la masificación (obstáculo inamovible al que se achacan todos los males), la objeción más común que nos suelen plantear los profesores de ciencias de universidad cuando se analiza con ellos un nuevo enfoque docente es que todo resulta muy bonito y perfecto en teoría, pero las cosas son muy diferentes en la práctica, por lo que lo razonable es no creerse nada hasta que no se vea que de verdad funciona. Por esta razón conviene tener a mano ejemplos creíbles y sólidos de situaciones o contextos reales parecidos a los que queremos abordar y que sirvan para demostrar que nuestras propuestas funcionan. Por ejemplo, si hablamos de una enseñanza basada en el método de proyectos o en la resolución de problemas (entendidos éstos en un sentido amplio), podemos citar el caso de la Facultad de Medicina de la Universidad McMaster, en Ontario, y el Worcester Polytechnical Institute, que fueron instituciones líderes en el uso de estas estrategias docentes (Campanario y Moya, 1999).

Un tema en el que siempre hemos observado un gran interés por parte de los profesores universitarios es el de la evaluación y calificación de los alumnos. Al contrario de lo que sucede con otras tareas docentes, la evaluación se deja exclusivamente en manos de los profesores y es responsabilidad total y absolutamente suya (Campanario, 1998b). En caso de fracaso, aquí no es posible el recurso a la queja, como puede suceder con la selección inicial de los alumnos que acceden a la universidad (que puede ser más o menos acertada), la distribución de alumnos por clases (con los típicos problemas de masi- ficación), las prácticas de laboratorio (que no siempre es posible programar para cuando los alumnos hayan estudiado la teoría) e incluso los libros de texto (que pueden ser más o menos adecuados).

Es muy difícil encontrar problemas y errores en la evaluación de los alumnos que puedan atribuirse a factores diferentes del profesor. Pues bien, no es raro descubrir fallos y deficiencias en los enunciados de los exámenes que se utilizan en la universidad (de hecho, es una tarea que hemos encargado a nuestros alumnos en cursos sobre técnicas de evaluación). Lo realmente interesante es que, en muchos casos, las deficiencias ayudan y benefician al alumno, algo que se pone de manifiesto especialmente en los exámenes de respuesta múltiple (Towns, y Robinson, 1993). Por ejemplo, un análisis de ítems en los exámenes de respuesta múltiple revela que, con frecuencia, algunos distractores erróneos son elegidos por una parte muy pequeña de los alumnos que contestan mal, lo cual, de hecho, implica que estos estudiantes no los tienen en consideración y que están recibiendo una ayuda involuntaria para aprobar. Es evidente que los profesores no están dispuestos a dejarse engañar por alumnos que aparentan saber y no saben y ésta es una ocasión para que surja la necesidad de evitar estos problemas y se plantee la oportunidad de intervenir.

Otro aspecto a tener en cuenta es que puede ser ventajoso dirigirnos a poblaciones heterogéneas, pero afines. Por ejemplo, puede ser interesante trabajar con profesores de química y de física porque el intercambio de experiencias puede ayudarnos a todos a darnos cuenta de que algunos problemas que creíamos exclusivos de nuestra disciplina, afectan también a otras. Además, este tipo de intercambio tal vez constituya una de las escasas ocasiones en las que profesores universitarios que trabajan en la formación de los mismos alumnos se reúnan a discutir problemas de aprendizaje de sus pupilos.

Aunque más arriba hemos señalado los peligros de que se nos confunda con defensores a ultranza del alumno, no hay que descartar el apoyo indirecto que podamos obtener de ellos. Este apoyo tiene que pasar por una sensibilización y formación de los estudiantes. Si éstos saben que existen soluciones y alternativas viables al problema de la docencia universitaria y se creen con la suficiente capacidad para exigir otros enfoques docentes, no cabe duda de que una parte de los profesores puede optar por implicarse en los necesarios procesos de formación. Es muy probable que, en el horizonte futuro, las universidades tengan que competir por los mejores alumnos, por lo que es de esperar que el mecanismo de la oferta y la demanda ponga un poco las cosas en su sitio.

Dado que, nos guste o no, la clase expositiva es y va a seguir siendo la estrategia docente dominante, tal vez haya que dedicar algún tiempo a tratar de mejorarla (Campanario, 2002). En cualquier caso, creemos que conviene insistir en la necesidad de sustituir progresivamente la clase expositiva por enfoques docentes más activos. En este empeño puede ser beneficioso trabajar en un orden inverso al que puede parecernos lógico a 
primera vista. Por ejemplo, como se ha indicado más arriba, la masificación sirve como excusa para seguir desarrollando clases expositivas. Sin embargo, es bastante común que, incluso en asignaturas optativas con pocos alumnos por aula, muchos profesores sigan empeñados en la clase expositiva (Plan Nacional de Evaluación de la Calidad de las Universidades, 2001). Por tanto, la disminución en el número de alumnos no garantiza necesariamente un cambio en las estrategias docentes. La inercia de tantos años es difícil de vencer si no existe una necesidad sentida de hacerlo así o una exigencia para el cambio. Tampoco facilita las cosas el no tener alternativas viables. Sin embargo, unas medidas sencillas de implementar tal vez ejerzan alguna presión sobre los profesores y consigan que éstos modifiquen sus estrategias docentes:

1) La petición generalizada para que los profesores proporcionen a los alumnos los apuntes de clase en CDROM o los hagan disponibles en internet. Aunque el coste sería irrisorio, es preciso articular los recursos necesarios para facilitar esta tarea. Si esto se hiciese así, es posible que muchos alumnos dejasen de acudir a las clases y decidiesen emplear ese tiempo en estudiar más que en copiar. Con ello la masificación disminuiría y se debilitaría una de las excusas para seguir desarrollando métodos tradicionales. Además, ¿qué sentido tendría dictar una información que ya tienen los alumnos?

2) La implantación progresiva de clases de dos horas. Esta medida casi desesperada está siendo considerada actualmente en Portugal para el nivel de enseñanza secundaria, y su objetivo es forzar a los profesores a hacer algo distinto de la clase expositiva, a la vez que se proporciona el necesario marco temporal para realizarlo (Caldeira, 2001). Esta medida sólo puede ser efectiva si se aplica de manera generalizada y no como una excepción.

La forma de abordar la formación de los profesores universitarios de ciencias también debería ser objeto de un debate. Probablemente lo primero que se nos ocurra sea organizar cursillos de capacitación, pero ésta no es la única alternativa posible ni, tal vez, deseable. Otra posibilidad consiste en trabajar directamente con los profesores, ayudándoles a articular sus clases, a seleccionar contenidos, a preparar materiales y a analizar los resultados de la evaluación. Por otra parte, puede resultar conveniente organizar seminarios o puestas en común en las que los profesores asistentes puedan plantear abiertamente sus problemas y dudas. Este planteamiento explícito de las dificultades como problemas puede servir para que los profesores aprecien mejor las ventajas de la necesaria formación didáctica. El correo electrónico brinda un medio potente que puede utilizarse para organizar y mantener listas de discusión entre los más interesados. Este recurso tiene la evidente ventaja de que permite a cada profesor dedicar a la tarea de formación el tiempo que quiera y cuando quiera.

Otra posibilidad consiste en la creación de centros de asistencia al profesor; por ejemplo, en la Universidad Autónoma de Madrid funciona un Servicio de Apoyo a la Docencia Universitaria (SADU), accesible desde la página web de la Universidad (www.uam.es). Una autora tan experimentada como Cruz propone incluso la creación de una agencia nacional para la formación pedagógica del profesorado universitario (Cruz, 2000, p. 31). Una alternativa que estamos tratando de implementar en nuestro departamento consiste en una página $w e b$ con recursos y sugerencias docentes.

Habría que pensar también en la elaboración de algunos materiales orientados específicamente a los profesores universitarios de ciencias. Estamos pensando, por ejemplo, en una breve recopilación de preguntas frecuentes sobre problemas de aprendizaje, organización de la docencia, alternativas o mejoras a la clase expositiva y a las prácticas de laboratorio tradicionales, etc. Se trataría de hacer algo parecido a un artículo que escribimos para la revista Tarbiya y en el que se revisaban los distintos aspectos relacionados con la evaluación que siempre suscitan dudas y aparecen de manera recurrente (Campanario, 1998b).

Por último, es evidente que todas las propuestas anteriores necesitan el respaldo de una actitud política decidida por parte de los equipos rectorales de las universidades correspondientes y de los departamentos universitarios. Por ejemplo, en los Estatutos de la Universidad de Alcalá se reconoce el papel de los departamentos como impulsores de la renovación pedagógica de sus profesores. Sin embargo, en el contexto actual, en general los profesores preocupados por la mejora de la calidad de su docencia «se sienten llaneros solitarios y forajidos» (Cruz, 2000, p. 26).

\section{DEBEMOS SER CONSCIENTES DE LOS RESULTADOS QUE PODEMOS ESPERAR}

A pesar de que la relación de problemas que hemos expuesto (algunos disimulados como oportunidades) es extensa, estamos obligados a ser optimistas. Dada la inexistencia de formación didáctica obligatoria para el profesorado de ciencias de universidad, casi cualquier cambio se va a traducir, probablemente, en una mejora en este aspecto.

De nuevo, un ejemplo tomado de la medicina nos ayudará a entender la situación. En muchos países empobrecidos, los problemas de salud son abrumadores. En este contexto, algunas medidas relativamente baratas, como pueden ser las vacunaciones masivas o el desarrollo de hábitos de higiene saludables (hervir agua, lavarse las manos antes de comer...) dan como resultado una mejora notable en la salud general. Igualmente, en el tema que abordamos, incluso cambios pequeños en las pautas docentes de los profesores universitarios de ciencias se pueden traducir en mejoras notables en la calidad de la docencia. Estas posibles mejoras, perceptibles a corto plazo, pueden dar mayor impulso a nuestros esfuerzos, a la vez que debilitan las argumentaciones y quejas de aquéllos que se resisten a cualquier novedad. Una vez abierta una brecha es más fácil derribar el muro. Por 
tanto, un logro que debemos valorar es que los posibles beneficiarios de los programas de capacitación didáctica no sólo sean protagonistas de un cambio, sino que se conviertan, en cierta medida, en propagandistas del mismo. Un medio para conseguir esta difusión puede ser dar a conocer los logros de los colectivos de docentes preocupados por la enseñanza de las ciencias en la universidad, y así tratar de incidir en otros grupos que a corto o mediano plazo podrían optar por incorporarse a esta tarea de transformación docente.

Además, debemos aprender de otras disciplinas. Así, por ejemplo, de nuevo, en medicina se sabe que muchos pacientes no van a seguir las recomendaciones de sus médicos y no van a tomar las dosis prescritas de medicamentos por diferentes razones que no vienen al caso. En esta tesitura podemos hacer dos cosas: lamentarnos de que nuestros pacientes no nos hagan caso o, como se hace en medicina, iniciar una línea de investigación y trabajo sobre los efectos de esta desobediencia.

Tal vez un objetivo modesto, pero útil, sea conseguir simplemente una $d u d a$ en el profesor acerca de la validez y adecuación de sus métodos docentes tradicionales de cara a mejorar el aprendizaje de sus alumnos. Aquí sucede algo parecido a lo que ocurre con nuestros alumnos. Muchas veces los estudiantes aprenden superficialmente y, por tanto, no saben que no saben y acuden a los exámenes convencidos de que dominan un contenido (Campanario, 2000). Hay poco que hacer con alguien que no sabe que tiene dudas porque, lógicamente, no es consciente de que necesita ayuda. En nuestro caso, tal vez un primer logro y no de los menores consista en plantear y suscitar dichas dudas en el profesor, referidas a su actuación docente. A partir de ahí, la necesidad puede traducirse en demanda y la demanda en capacitación profesional.

Por otra parte, puede que nos resulte difícil conseguir en la universidad un número elevado de una de las figuras estelares de la didáctica de las ciencias: el profesorinvestigador, dado que nuestros beneficiarios ya están bastante ocupados con sus propias tareas de investigación científica seria. Esta situación no debería desanimarnos: al igual que el ingeniero puede construir ordenadores muy potentes sin estar necesariamente al tanto de las implicaciones filosóficas de la mecánica cuántica en que se basan los chips que utiliza, así, el profesor de universidad puede beneficiarse de muchos conocimientos y destrezas sin necesidad de someterse al lento proceso de formación necesario para comprender las bases de nuestra área de conocimientos.

Por último, cabe citar un resultado previsible indirecto, pero de indudable valor: el efecto multiplicador que una formación pedagógica del profesorado universitario tendría en otros niveles. Como se ha señalado más arriba, los futuros profesores aprenden a enseñar fundamentalmente durante su largo proceso de enculturación como estudiantes universitarios (Campanario, 1998a). Si estos futuros profesores ven hacer algo más que impartir clases expositivas o se enfrentan a otro tipo de trabajo de laboratorio, más parecido a una verdadera investigación científica que a una receta de cocina, no cabe duda de que tratarán de reproducir estas estrategias en sus respectivos niveles cuando accedan a la profesión docente.

\section{PARA FINALIZAR: ALGUNAS CONCLU- SIONES Y PERSPECTIVAS}

Si bien la tarea que debemos abordar es ambiciosa y difícil, no es imposible. Hoy día el área de didáctica de las ciencias experimentales ha alcanzado un desarrollo notable y se articula como una más de las que existen en la universidad española. Como señala Gil, a comienzo de la década de los ochenta el panorama en didáctica de las ciencias era pobre: «[...] no existían revistas españolas que pudieran servir de comunicación e impulso y las publicaciones internacionales eran desconocidas, las facultades de ciencias rechazaban, o simplemente ignoraban, los problemas educativos como tema de investigación y elaboración de tesis [...] no se conocía ningún equipo -más allá de algunas individualidades aisladascon dedicación sistemática al desarrollo de dicha investigación» (Gil, 1994, p. 154). El cambio ha sido notable. Así, según la página web del Ministerio de Educación y Ciencia (www.mec.es), actualmente hay 222 profesores del área (9 CU, 29 TU, 57 CEU y 127 TEU). Según Sánchez, Pro y Valcárcel (1997, p. 35), «factores como la institucionalización de los correspondientes departamentos universitarios, la proliferación de trabajos de investigación, la aparición de tesis doctorales, la difusión de los resultados en revistas especializadas y la comunicación de experiencias en reuniones de profesores y especialistas, han contribuido a dar un impulso notable a este ámbito del conocimiento».

A la vista de lo anterior parece que lo más difícil (poner en marcha el área) ya está hecho. Ahora nuestra tarea consiste en conseguir ocupar todos los espacios que creemos que nos pertenecen y en creernos que tenemos el derecho a ello y el deber de hacerlo. Si no abordamos el reto por nuestra cuenta, otras áreas de conocimiento más generales ocuparán este terreno y luego será difícil conseguir que nos dejen intervenir. Tal vez haya llegado el momento de que los que trabajamos en el área de didáctica de las ciencias nos quitemos de encima un cierto complejo de inferioridad que, seguramente, nos impide avanzar más allá de donde estamos. Trabajamos en un terreno excitante desde el que podemos influir directamente en las personas, tenemos que recurrir a varias disciplinas que dan sentido y sirven de fundamentación en nuestro campo de conocimientos y actuación y estamos participando en unos momentos históricos en los que está cuajando un nuevo dominio ¿Por qué privar a otros de las ventajas (y placeres) que se derivan inevitablemente de estas dinámicas?

Ha llegado el momento de plantear en la agenda del área para el siglo xxi la necesidad de abordar el asalto a una fortaleza que, aunque parece inexpugnable, tiene, como toda alcazaba, resquicios, túneles, puertas falsas, desniveles y peraltes que ofrecen una guía a un asaltante imaginativo o a un ejército audaz. 


\section{AGRADECIMIENTOS}

Quiero dar las gracias a María Mercedes Callejas por introducirme en el arduo y apasionante problema de la formación didáctica del profesorado universitario. También quiero agradecer

\section{REFERENCIAS BIBLIOGRÁFICAS}

ALMAJANO, M.P. y VALERO-GARCÍA, M. (2000). El PROFI: Programa de formación inicial del ICE de la UPC. Revista Interuniversitaria de Formación del Profesorado, 38, pp. 67-78.

BELÉNDEZ, A. (1996) Algunas consideraciones en torno al proceso enseñanza-aprendizaje de la física en la universidad. Revista Interuniversitaria de Formación del Profesorado, 27, pp. 189-203.

BLÁZQUEZ, F. (1999). Una experiencia española: El plan de formación del ICE de la Universidad de Extremadura. Revista Interuniversitaria de Formación del Profesorado, 34, pp. 171-185.

CALATAYUD, M.L. y GIL, D. (1993). La preparación docente del profesorado de facultades de ciencias: Una necesidad emergente. Enseñanza de las Ciencias, núm. extra, pp. $35-36$.

CALDEIRA, M.H. (2001) Comunicación personal (Mayo, 2001)

CAMPANARIO, J.M. (en revisión). Contra algunas concepciones y prejuicios comunes de los profesores universitarios de ciencias sobre la didáctica de las ciencias.

CAMPANARIO, J.M. (1998a) ¿Quienes son, qué piensan y qué saben los futuros maestros y profesores de ciencias?: Una revisión de estudios recientes. Revista Interuniversitaria de Formación del Profesorado, 33, pp. 121-140.

CAMPANARIO, J.M. (1998b) Preguntas y respuestas sobre la evaluación de los alumnos en la enseñanza de las ciencias. Tarbiya: Revista de Investigación e Innovación Educativa, 19, pp. 69-84.

CAMPANARIO, J.M. (2000) El desarrollo de la metacognición en el aprendizaje de las ciencias: estrategias para el profesor y actividades orientadas al alumno. Enseñanza de las Ciencias, 18(3), pp. 369-380.

CAMPANARIO, J.M. (2002) Algunas sugerencias concretas para mejorar las clases magistrales de ciencias incluso en aulas masificadas. Docencia Universitaria (en prensa, aceptado para su publicación).

CAMPANARIO, J.M. y MOYA, A. (1999) ¿Cómo enseñar ciencias? Las principales tendencias y propuestas. Enseñanza de las Ciencias, 17(2), pp. 179-192.

CARTAÑA, J. y COMÁS, M.C. (1994) Algunas consideraciones alrededor de la concepción constructivista de las ciencias experimentales: Dualidad entre ciencia y su enseñanza. $L a$ didáctica de las ciencias experimentales a debate. Universidad de Murcia: Murcia.

CRUZ, M.A. (1993). La formación inicial para la docencia universitaria. Tarbiya, 4 , pp. 65-88.

CRUZ, M.A. (2000). Formación pedagógica inicial y permanente del profesor universitario en España: Reflexiones y propuestas. Revista Interuniversitaria de Formación del Profesorado, 38, pp. 19-35. los comentarios y sugerencias de dos asesores anónimos de la revista.

CRUZ, M.A. y GRAD, H. (1993). Los programas de formación inicial para la docencia universitaria en la Universidad Autónoma de Madrid. Tarbiya, 4, pp. 91-107.

ESCUDERO, J.M. (1999). La formación permanente del profesorado universitario: Cultura, política y procesos. Revista Interuniversitaria de Formación del Profesorado, 34, pp. 133-157.

GIL, D. (1993). Contribución de la historia y de la filosofía de las ciencias al desarrollo de un modelo de enseñanza-aprendizaje como investigación. Enseñanza de las Ciencias, 11(2), pp. 197-212.

GIL, D. (1994). Diez años de investigación en didáctica de las ciencias. Realizaciones y perspectivas. Enseñanza de las Ciencias, 12(2), pp. 154-164.

GIL, D., CARRASCOSA, J., FURIÓ, C. y MARTÍNEZTORREGROSA, J. (1991). La enseñanza de las ciencias en la educación secundaria. Barcelona: ICE, Universitat de Barcelona.

GIL, D., MARTÍNEZ-TORREGROSA, J. y SENENT, F. (1988) El fracaso en la resolución de problemas de física: Una investigación orientada por nuevos supuestos. Enseñanza de las Ciencias, 6(2), pp. 131-146.

GIL, D. y VILCHES, A. (1999). Problemas de la educación científica en la enseñanza secundaria y la universidad: contra las evidencias. Revista Española de Física, 13(5), pp. 10-15.

MELLADO, V.(1999). La formación didáctica del profesorado universitario de ciencias experimentales. Revista Interuniversitaria de Formación del Profesorado, 34, pp. 231-241.

MELLADO, V. y CARRACEDO, D. (1993). Contribuciones de la filosofía de la ciencia a la didáctica de las ciencias. Enseñanza de las Ciencias, 11(3), pp. 331-339.

PERALES, F.J. y CAÑAL DE LEÓN, P. (2000). Didáctica de las ciencias experimentales. Compilación. Alcoy: Marfil.

PLAN NACIONAL DE EVALUACIÓN DE LA CALIDAD DE LAS UNIVERSIDADES (2001). Licenciado en química. Autoinforme de evaluación Universidad de Alcalá de Henares: Alcalá de Henares.

PORLÁN, R. (1998). Pasado, presente y futuro de la didáctica de las ciencias. Enseñanza de las Ciencias, 16(1), pp. 175-185.

SÁNCHEZ, G., PRO, A. y VALCÁRCEL, M.A.V. (1997). La utilización del modelo de planificación de unidades didácticas: El estudio de las disoluciones en la educación secundaria. Enseñanza de las Ciencias, 15(1), pp. 35-50.

TOWNS, M.H. y ROBINSON, W.R. (1993). Student use of test-wiseness strategies in solving multiple-choice chemistry examinations. Journal of Research in Science Teaching, 30, pp. $709-722$.

[Artículo recibido en junio de 2001 y aceptado en diciembre de 2001.] 